\title{
SOD1 Transcriptional and Posttranscriptional Regulation and Its Potential Implications in ALS
}

\author{
Pamela Milani, ${ }^{1,2}$ Stella Gagliardi, ${ }^{1}$ Emanuela Cova, ${ }^{1}$ and Cristina Cereda ${ }^{1}$ \\ ${ }^{1}$ Laboratory of Experimental Neurobiology, IRCCS, National Neurological Institute “C. Mondino," Via Mondino 2, 27100 Pavia, Italy \\ ${ }^{2}$ Department of Neurological Sciences, University of Pavia, 27100 Pavia, Italy
}

Correspondence should be addressed to Pamela Milani, pame.milani@gmail.com

Received 15 November 2010; Accepted 3 February 2011

Academic Editor: Prabhakara V. Choudary

Copyright () 2011 Pamela Milani et al. This is an open access article distributed under the Creative Commons Attribution License, which permits unrestricted use, distribution, and reproduction in any medium, provided the original work is properly cited.

\begin{abstract}
Copper-zinc superoxide dismutase (SOD1) is a detoxifying enzyme localized in the cytosol, nucleus, peroxisomes, and mitochondria. The discovery that mutations in SOD1 gene cause a subset of familial amyotrophic lateral sclerosis (FALS) has attracted great attention, and studies to date have been mainly focused on discovering mutations in the coding region and investigation at protein level. Considering that changes in SOD1 mRNA levels have been associated with sporadic ALS (SALS), a molecular understanding of the processes involved in the regulation of SOD1 gene expression could not only unravel novel regulatory pathways that may govern cellular phenotypes and changes in diseases but also might reveal therapeutic targets and treatments. This review seeks to provide an overview of SOD1 gene structure and of the processes through which SOD1 transcription is controlled. Furthermore, we emphasize the importance to focus future researches on investigating posttranscriptional mechanisms and their relevance to ALS.
\end{abstract}

\section{Introduction}

$\mathrm{Cu} / \mathrm{Zn}$ superoxide dismutase or SOD1 is a soluble protein acting as a $32 \mathrm{kDa}$ homodimeric enzyme to convert naturally occurring, but harmful, superoxide radicals to molecular oxygen and hydrogen peroxide. SOD1 is one of the three human superoxide dismutases identified and characterized in mammals: copper-zinc superoxide dismutase $(\mathrm{Cu} / \mathrm{ZnSOD})$, manganese superoxide dismutase (MnSOD or SOD2), and extracellular superoxide dismutase (ECSOD or SOD3) [1]. When SOD1 was isolated for the first time, it was thought to be a copper storage protein [2]; the catalytic function of SOD1 was discovered in 1969 by McCord and Fridovich [3], and it was clear that SOD1 acts as a scavenger of superoxide, through a two-step reaction involving reduction and reoxidation of the copper ion in its active site [4]. Primarily, this reaction occurs in the cytoplasm where SOD1 is highly expressed. However, immunohistochemical analysis in rat hepatocytes identified SOD1 in other different subcellular organelles, such as nucleus, lysosomes, and mitochondria [5]. In the 1990s, the scientific community focused their studies on the genetic and biochemical characterization of SOD1 [6], demonstrating that SOD1 plays an important role in diseases as heart failure [7], cancer [8], diabetes [9], Down's syndrome [10], and amyotrophic lateral sclerosis [11]. In fact, in 1984, the first paper about Down's Syndrome was published [12], and in 1993, the first SOD1 gene mutations associated with ALS were described [11]. In this paper, we focused on SOD1 gene structure and organization, transcriptional and post-transcriptional gene regulation, and their contributions in the pathogenesis of ALS.

\section{Gene Structure and Organization}

The human SOD1 gene (Entrez Gene ID 6647) is located on chromosome 21q22.11, and it codes for the monomeric SOD1 polypeptide (153 amino acids, molecular weight $16 \mathrm{kDa}$ ). More precisely, this gene is located from base pair $33,031,935$ to base pair $33,041,241$ with a genomic size of $9307 \mathrm{bp}$, according to UCSC Genome Browser (GRCh37/ hg19; http://genome.ucsc.edu/). The coding region consists of five exons interrupted by four introns (Figure 1). Several polymorphisms have been identified in SOD1 gene, mainly distributed in the regulatory regions, including promoter, 


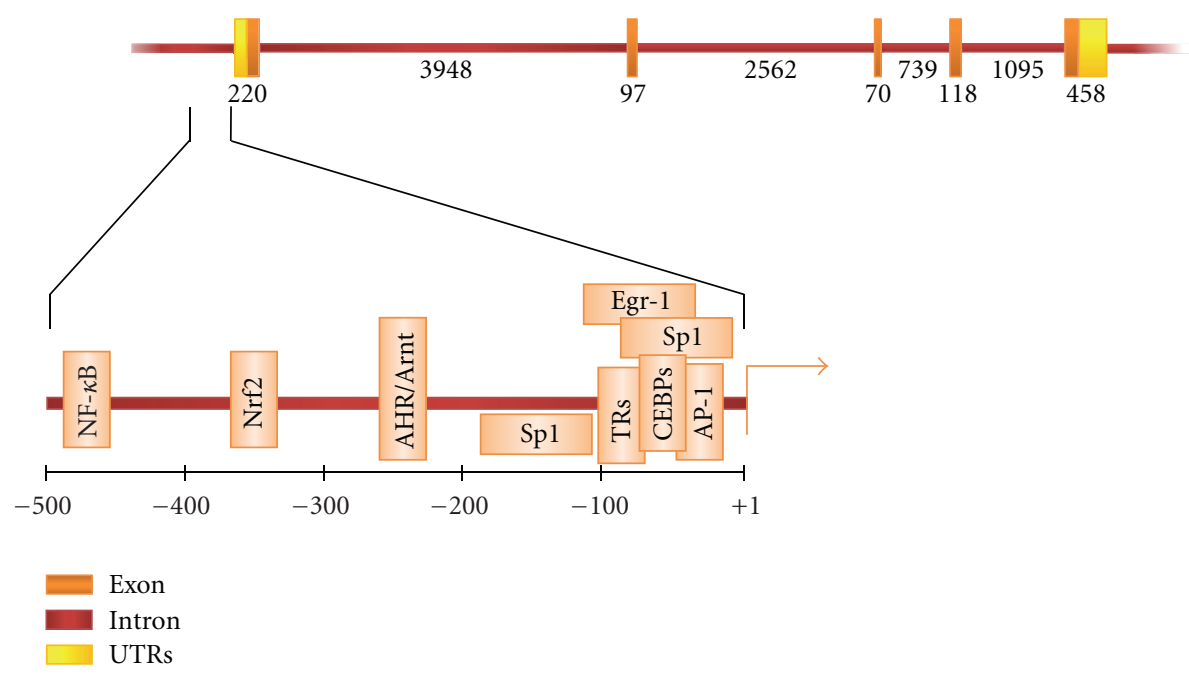

FIGURE 1: Genomic organization of human SOD1 gene. The size of exons and introns, in base pairs, is shown in association with each fragment. The $5^{\prime}$ flanking region is expanded, and the transcription factors, interacting with the corresponding DNA regulatory elements, are shown at the bottom. The transcription start site is depicted as an arrow at position +1 .

UTRs, and introns. With regards to SOD1 mRNA splicing, it has been demonstrated that the donor sequence at the first intron presents a $\mathrm{T}$ to $\mathrm{C}$ variant and, consequently, it deviates from the highly conserved $5^{\prime}$ GT... AG $3^{\prime}$ consensus sites. Nevertheless, it has been demonstrated that this unconventional splice junction ( $5^{\prime}$ GC... AG $3^{\prime}$ ) is functional [13].

The proximal promoter of human SOD1 gene, involved in the basal transcription, has been well studied, and it contains not only the TATA box, but also a CCAAT box and a GC-rich region, which are recognized by CCAAT/enhancerbinding proteins (C/EBPs) and specificity protein $1(\mathrm{Sp} 1)$, respectively [14]. Other binding sites for Egr1, AP1, AHR, $\mathrm{Nrf} 2, \mathrm{NF}-\kappa \mathrm{B}$, and TR transcription factors have been also identified and verified by functional studies (Figure 1). These transcription factors are involved in the regulation of SOD1 inducible gene expression under specific extra- and intracellular conditions. Different studies performed in rat cellular lines have identified several other regulatory sequences in the rat SOD1 promoter [15-18]. Considering that the rat SOD1 gene is very similar to that of human, especially with regard to the proximal part of the promoter region [18], further efforts will be necessary to identify possible other regulatory elements in the human promoter. Moreover, in view of the fact that regulatory elements can be located up to several hundred kilobases away from the gene they control, experiments should be carried out to test whether the longrange control of transcription may represent a mechanism involved in human SOD1 gene expression.

With regard to the $5^{\prime}$ untranslated region ( $5^{\prime}$ UTR), Sherman and colleagues [19] demonstrated that SOD1 mRNA posseses various $5^{\prime}$ termini, which are mapped by both primer extension and Sl mapping. The authors showed that the vast majority of the mRNA species has a $5^{\prime}$ start site located 23 bp downstream the TATA box (TATAAA), while the other mRNA molecules have $5^{\prime}$ termini 30,50 , and
$65 \mathrm{bp}$ upstream from the major transcription start site. Consequently, these mRNA species are produced through TATA-independent transcription. It would be interesting to perform in-depth investigations about the functional relevance of these multiple transcription start sites, mainly to determine the potential cell and tissue specificity of the different mRNA species.

Furthermore, two SOD1 mRNAs of about $0.7 \mathrm{~kb}$ and $0.9 \mathrm{~kb}$ have been identified in a variety of cells, and it has been shown that they are transcribed from the same gene and differ in the length of their $3^{\prime}$ UTRs caused by multiple polyadenylation sites. Indeed, analysis of the DNA sequence at the $3^{\prime}$ UTR region of the SOD1 gene revealed the presence of two groups of processing/polyadenylation signals; the first one contains two signals (AATAAA and ATTAAA). The former is fused to the terminal portion of the coding region and it is not utilized. On the contrary, the second site (ATTAAA), localized $76 \mathrm{bp}$ downstream the stop codon, is the one involved in the production of the $0.7 \mathrm{~kb}$ mRNA [13] (Figure 2). The second group includes three polyadenylation signals, located $200-250 \mathrm{bp}$ further downstream; the middle one (AATAAA) is involved in the formation of the $0.9 \mathrm{~kb}$ SOD1 mRNA (Figure 2). Both the mRNA species are functional, since they can be translated in vitro to immunoprecipitable SOD1 proteins $[19,20]$. The longer transcript is approximately four times less abundant than the $0.7 \mathrm{~kb}$ mRNA.

\section{Transcriptional Factors Involved in SOD1 Constitutive and Inducible Expression}

Even if SOD1 has been often considered a "housekeeping gene" due to its high and ubiquitous expression, it is now clear that its induction is fine-tuned modulated by complex intracellular events which probably involve multiple positive and negative regulatory elements acting in concert. Indeed, 


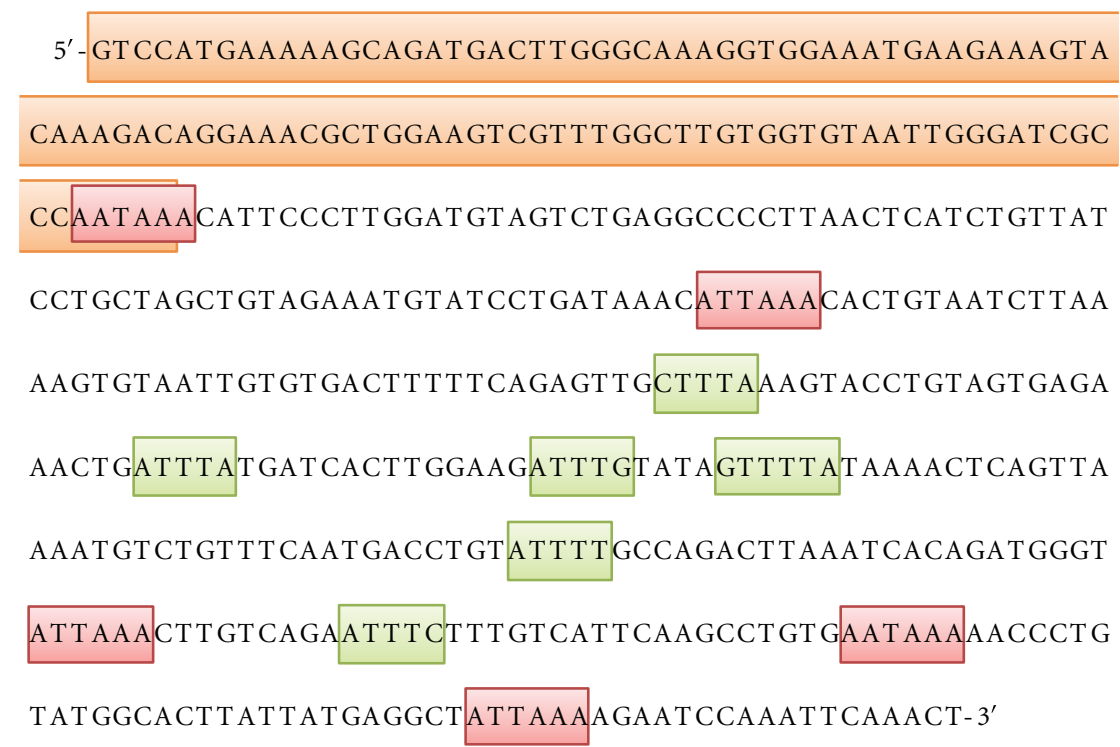

FIGURE 2: Nucleotide sequence of the fifth exon of SOD1 gene. The coding portion is highlighted in orange; the polyadenylation sites are in red, and the A/U-rich elements are in green.

the diversity of SOD1 inducers means that there are multiple cis-acting elements for this gene, and many studies have been performed to precisely identify the location and the functional relevance of both these DNA sequences and the corresponding trans-acting factors.

3.1. C/EBPs (CCAAT/Enhancer Binding Proteins). These proteins are a family of transcription factors, all containing a highly conserved, basic leucine zipper (bZIP) domain at the $\mathrm{C}$-terminus. It has been demonstrated that C/EBP-related factors are necessary for SOD1 constitutive expression; both $\mathrm{C} / \mathrm{EBP} \alpha$ and $\mathrm{C} / \mathrm{EBP} \beta$ can interact with the CAAT box (located at position -64 to -55 from transcription start site), playing similar and nonmutually exclusive roles on SOD1 basal transcription [21]. This C/EBP consensus element partially overlaps the Sp1/Egr1 sequence, suggesting that an interlaced network among these transcription factors may act in the fine control of regulation of SOD1 gene expression.

Furthermore, it has been recently demonstrated that also the transcription factor CCAAT/enhancer binding protein delta (CEBPD, $\mathrm{C} / \mathrm{EBP} \delta$, NF-IL6 $\beta$ ) is involved in the regulation of human SOD1 transcription [22]. Specifically, CEBPD enhances SOD1 mRNA expression in cisplatintreated human urothelial carcinoma cell line (NTUB1) via direct promoter transactivation.

3.2. Sp1 (Specificity Protein 1). Sp1 is a ubiquitously expressed $\mathrm{C} 2 \mathrm{H} 2$-type zinc finger-containing DNA binding protein. It binds GC-rich motifs (such as $5^{\prime}-\mathrm{G} / \mathrm{T}-\mathrm{GGGCGG-}$ G/A-G/A-C/T-3' or 5'-G/T-G/A-GGCG-G/T-G/A-G/A-C/T$\left.3^{\prime}\right)$ with high affinity and enhances transcription with one of the two glutamine-rich domains [23]. It has been demonstrated that overexpression of Sp1 conspicuously enhances SOD1 basal promoter activity [24].

3.3. Egr1 (Early Growth Response-1). Egr1 is a nuclear phosphoprotein of $80 \mathrm{kDa}$ that functions as a regulator of transcription and belongs to the family of early response genes; it is rapidly induced by mitogens to transduce the proliferative signal. It has also been demonstrated that cytokines and stress signals such as radiation, injury, and oxidative or mechanical stress can induce the expression of this transcription factor [25-29]. In a paper published by Minc and coworkers [30], it has been demonstrated that SOD1 mRNA level is rapidly increased after the treatment of HeLa cells with phorbol-12-myristate-13-acetate (PMA), and the region between nucleotides -59 and -48 has been identified as the one responsible for PMA-induced expression. This region presents noncanonical consensus recognition sequences for Sp1 and Egr-1, and it is bound by Sp1 in a constitutive manner and by Egr1 in response to PMA exposure.

3.4. AP1 (Activating Protein 1). AP-1 is a homo- or heterodimeric transcription factor made by proteins from Jun, Fos, and Maf subfamilies. Activating transcription factor (ATF) proteins also belong to AP1. All these proteins are basic leucine zipper (bZIP) transcription factors. The activity of AP-1 proteins can be regulated by a broad range of environmental cues, including growth factors, cytokines, and oxidative stress, which initiate a variety of intracellular pathways to transduce the information from the extracellular milieu to the nuclear compartment, thus leading to specific cellular responses.

It has been demonstrated that AP1 represses SOD1 transcription by sequestrating essential coactivators, such as $\mathrm{Sp} 1$, rather than interacting directly with SOD1 gene promoter [24].

Furthermore, in agreement with the previous results demonstrating AP1 transcriptional repression activity, it has been shown that neuronal nitric oxide synthase (nNOS) over-expression causes the downregulation of SOD1 in terms of mRNA, protein, and activity levels [31] and that this is caused by two events: the decreased binding of $\mathrm{Sp} 1$ to 
SOD1 promoter, caused by nNOS interaction with $\mathrm{Sp} 1$, and a concomitant increased binding activity of AP1 to the same site.

3.5. AHR (Aryl Hydrocarbon Receptor). AHR is a ligandactivated transcription factor belonging to the helix-loophelix (bHLH) family. It is well known that, prior to ligand binding, AHR exists in a latent state in the cytosol associated with HSP90 and HSP90 accessory proteins [32-34]. The interaction with HSP90 is fundamental to retain AHR in the cytoplasm. Many synthetic halogenated and nonhalogenated aromatic hydrocarbons activate AHR signal pathway through a direct interaction: upon ligand binding, HSP90-bound AHR translocates into the nucleus where it exchanges HSP90 for another bHLH protein, known as hydrocarbon receptor nuclear translocator (Arnt). This new heterodimeric complex binds to the xenobiotic responsive element (XRE) that functions as a cis-acting enhancer in the promoter region of numerous phase I and II drug-metabolizing enzyme genes $[35,36]$. Cho and coworkers [37] observed an increased expression of SOD1 mRNA and protein after the exposure of human HepG2 and HeLa cells to one of the most toxic man-made hazard, the 2,3,7,8-tetrachlorodibenzo-pdioxin (TCDD), an environmental contaminant belonging to the halogenated aromatic hydrocarbons class and interacting with AHR. The authors identified the presence of a xenobiotic responsive element in the 5 flanking region of human SOD1 gene (located between -255 and -238 from the transcription start site), which is responsible for the induction by TCDD.

3.6. Nrf2 (Nuclear Factor E2-Related Factor 2). Nrf2 is a Cap'n' collar (Cnc) transcription factor that regulates the expression and the coordinated induction of a battery of defensive genes encoding phase II detoxifying enzymes and antioxidant proteins. The activity of Nrf2 is controlled by the cysteine-rich cytosolic INrf2 (Inhibitor of Nrf2), also known as Keap1 (Kelch-like ECH-associated protein1). The activation of Nrf2 pathway requires its cytosolic stabilization via oxidative modification of distinct Keap1 cysteine residues, Keap1 preoteasomal degradation, and/or phosphorylation of Nrf2. The dissociation from Keap1 is a prerequisite for Nrf2 translocation to the nucleus. In this compartment, Nrf2 heterodimerizes with a small Maf protein and binds to the cis-acting antioxidant/electrophile responsive element (ARE/EpRE), activating the transcription of various cytoprotective genes involved in detoxification from xenobiotics, electrophile conjugation, ROS scavenging, and regulation of intracellular redox homeostasis [38-41].

An antioxidant responsive element (located between -356 and -330 from the transcription start site) has been identified in human SOD1 gene promoter [42, 43]. Park and colleagues demonstrated that $S O D 1$ gene transcription is induced in human HepG2 hepatoma cells after the treatment with the dioxin TCDD, which produces reactive oxygen species thus leading to the activation of Nrf2 signalling. Moreover, it has been shown that low-dose and nontoxic proteasome inhibition enhances mRNA and protein expression of SOD1 in different human endothelial and vascular smooth muscle cells through transcriptional induction mediated by Nrf2 [43].

3.7. NF- $\kappa B$ (Nuclear Factor-KappaB). The term NF- $\kappa \mathrm{B}$ refers to a family of five structurally related transcription factors (p50, p52, RelA/p65, c-Rel, and RelB), all containing the Rel homology domain (RHD) within the N-terminus and acting as homo- and heterodimeric DNA binding complexes [44]. Their functionality and nuclear localization are controlled by a family of inhibitor proteins, known as IkappaBs $(\mathrm{I} \kappa \mathrm{Bs})$. In nonstimulated cells, NF- $\kappa \mathrm{B}$ dimers are bound to inhibitory $\mathrm{I} \kappa \mathrm{B}$ proteins and are thereby sequestered in the cytoplasm as inactive complexes. Several studies showed that NF- $\kappa \mathrm{B}$ activity is induced in most cell types in response to a broad variety of stimuli, ranging from cytokines, radiation, and oxidative stress (such as exposure to $\mathrm{H}_{2} \mathrm{O}_{2}$ ), with major roles in coordinating innate and adaptive immunity, cell activation and proliferation, survival, development, and apoptosis [45, 46]. NF- $\kappa \mathrm{B}$ was one of the first transcription factors shown to be redox regulated [47-50], and Rojo and colleagues [51] showed that cell treatment with $\mathrm{H}_{2} \mathrm{O}_{2}$ initiates the PI3K/Akt cascades, which participates in NF- $\kappa \mathrm{B}$ activation and in subsequent SOD1 transcriptional induction. Indeed, the authors identified a p65-NF- $\kappa \mathrm{B}$ binding site in the human SOD1 promoter (GGTAAGTCCC), and they demonstrated that Akt-activated NF- $\kappa \mathrm{B}$ presents increased binding to this sequence, mediating the upregulation of SOD1 expression.

3.8. TRs (Thyroid Hormone Receptors). The thyroid hormone receptors are encoded by the TRalpha and TRbeta genes and are ligand-dependent transcription factors, since they bind both thyroid hormones (THs) and TH-response elements (TREs) that are located in the promoters of target genes. These proteins, which belong to the nuclear receptor superfamily, regulate development and a broad variety of critical cellular functions including growth, differentiation, basal metabolic rate, and metabolism of protein, fat, and carbohydrate. It has been demonstrated that TRs can either enhance or repress transcription. Hormonedependent repression requires binding to negative TREs (nTREs); in particular, the unoccupied receptor increases transcription on nTREs, and ligand binding to TR reverses this induction [52]. The responsive sites of these negatively regulated genes are generally localized to the proximal promoter region [53]. In agreement with this observation, Santos and co-workers [54] have identified a thyroid hormone inhibitory element between -157 and +17 of the human SOD1 promoter and demonstrated that $\mathrm{T}_{3}$ exposure reverses the induction of SOD1 transcription caused by the ROS-producing paraquat and PMA agents, through the direct $\mathrm{TR} / \mathrm{T}_{3}$-DNA interaction. On the contrary, SOD1 promoter is significantly upregulated by unliganded TRs.

\section{Posttranscriptional Gene Regulation}

Historically, efforts aimed at decoding the molecular mechanism of gene expression have been principally focused on 
transcriptional control. However, post-transcriptional regulation of mRNAs is now considered as an important step in the flow of genetic information providing additional opportunities by which gene expression could be rapidly modulated. Indeed, post-transcriptional events such as mRNA processing and nuclear export, mRNA stability, translational efficiency, and microRNA-dependent modulation create a complex intracellular network contributing to determine the global levels of specific mRNAs. Most mRNA regulatory elements are located within the $5^{\prime}$ and $3^{\prime}$ UTRs, where they function as platforms for the binding of numerous proteins and noncoding RNAs. The $5^{\prime}$ UTR is principally involved in controlling mRNA translation [55], while the $3^{\prime}$ UTR regulates multiple steps of mRNA metabolism and stability.

Very few works on post-transcriptional regulation of SOD1 have been published so far.

As mentioned above, two species of SOD1 mRNA with different $3^{\prime}$ UTR lengths have been identified, and they produce in vitro different quantities of SOD1 protein. Kilk and colleagues [56] performed functional study to investigate the effect of this regulatory region on SOD1 protein expression, and they observed that cells transfected with a long cDNA, containing the long $3^{\prime} \mathrm{UTR}$, produce three times more SOD1 protein than cells transfected with a cDNA presenting a deletion of the last $185 \mathrm{bp}$ from the 3'UTR. The authors hypothesized that the ability of the long mRNA to produce more SOD1 enzyme may depend on specific sequences located in the $3^{\prime}$ UTR, and they identified the presence of various $\mathrm{A} / \mathrm{U}$-rich elements (AREs) in this region (AUUUA, CUUUA, AUUUG, GUUUUA, AUUUU, and AUUUC) (Figure 2). AREs were initially defined by the sequence AUUUA [57], although subsequent studies showed other sequences with U-stretches presenting the same properties as the canonical element. Several AREbinding proteins, which function as trans-acting factors, have been identified, and interaction of these proteins can correlate negatively or positively with the stability and the translatability of the target mRNA [58]. Considering the biological relevance of these sequences, the identification of the proteins potentially interacting with SOD1 mRNA may shed new light on SOD1 gene expression modulation. Moreover, another important question is whether these SOD1 mRNA species, differing for their $3^{\prime}$ UTR lengths, could be somehow linked to pathological conditions, since it is possible that specific cellular stresses may vary the relative proportions of such variants with relevant implications for cell phenotypes.

In addition, also SOD1 post-transcriptional regulation mediated by microRNAs (miRNAs) represents a field still almost unexplored. miRNAs, which are small non-proteincoding RNAs, function as key post-transcriptional regulators of gene expression by usually base pairing to the $3^{\prime}$ UTR of the target mRNAs to cause translational repression and mRNA decay $[59,60]$. Recently, Wang and co-workers [61] showed, through computational and biological approaches, that SOD1 is a target of miR-377 in human and mouse mesangial cells and miR-377 diminished SOD1 protein levels. Owing to the importance of miRNAs in mRNA metabolism control, future in-depth researches should be carried out in this field to unravel previously unrecognized complex regulatory and interactive pathways that may cooperate to modulate SOD1 quantities and whose dysregulation may be relevant for ALS disease states.

\section{Amyotrophic Lateral Sclerosis}

Amyotrophic lateral sclerosis disease (ALS) is a multifactor and multigenic disorder with still unknown aetiology and pathogenesis. Even if several new genes associated to ALS have been described, SOD1 gene is considered the major gene involved in ALS pathogenesis.

5.1. Genetic Variants. In 1991, Siddique and collaborators [62] identified a linkage of familial ALS to the SOD1 locus on chromosome 21q22 and demonstrated genetic locus heterogeneity in FALS studying 23 ALS families. In 1993, Rosen [11] and collaborators have reported tight genetic linkage between ALS and SOD1 gene, establishing SOD1 as the first causative gene for ALS (genetic nomenclature, ALS1). Mutations in SOD1 gene are responsible for $12-$ $23 \%$ of all FALS cases [63, 64]. More than 150 SOD1 mutations have been reported in 68 of the 153 codons, spread over all five exons (ALS Online Genetic Database, ALSOD: http://alsod.iop.kcl.ac.uk/) [65], most of them cause disease. Although most mutations are missense, nonsense mutations and deletions have also been found $[66,67]$. SOD1 exonic mutations have occasionally been described in patients with apparently sporadic onset, and it has been estimated that $1 \%$ of SALS could be due to SOD1 mutations [68]. Many of the mutations are at present unique to individual families. Correlation between mutations and phenotype has been investigated, because of a large variability in phenotype in term of disease progression, extramotor features, and age of onset but is generally difficult to predict on the basis of the SOD1 mutations [66].

5.2. SOD1 Gene Expression in ALS. Even if SOD1 gene has been considered fundamental in ALS, numerous studies have been done concerning protein expression and gene mutations, but mRNA level studies are very rare. For the first time, in 1997, Nishiyama studied SOD1 expression in cervical and spinal cord of ALS patients using a quantitative in situ hybridization technique [69]. There were no significant differences between the amounts of SOD1 mRNA level observed in patients with sporadic or familial disease, and normal control subjects. Moreover, motoneurons in the normal spinal ventral horn and precentral motor cortex exhibited significantly higher levels of SOD1 messenger RNA than did other neurons [69]. In recent years, expression studies often produced conflicting data about up- or downregulation of ALS relevant genes, such as SOD1 [70-72]. The confounding data were probably due to differences in the central nervous system (CNS) tissue areas analyzed and possibly differences in the analytical techniques used.

The latest SOD1 gene expression study demonstrated that SOD1 mRNA level is elevated in specific nervous areas typically affected by ALS disease (i.e., brain stem and 
spinal cord) and not in other brain areas not involved in the neurodegenerative process (i.e., cerebellum and cerebral cortex) in SALS patients [73]. Moreover, increased SOD1 mRNA expression has been detected in peripheral system as lymphocytes from SALS patients compared to healthy people [73]. However, in the same work, Western Blotting analysis showed lower or similar expression of the protein both in lymphocytes and in nervous tissue-affected areas thus confirming a previous observation obtained in the peripheral cells [74]. On the contrary, histopathological analysis of spinal cord tissue from SALS patients evidenced an increased SOD1 protein expression compared with controls. This result correlates with the evidence of higher mRNA level and suggests the hypothesis that, in at least a subgroup of SALS patients, misfolded and aggregated SOD1 protein would precipitate in the insoluble fraction becoming undetectable after extraction with routinely used lysis buffers. Actually, proteinaceous inclusion bodies that may contain SOD1 have been already described in motor neurons of SALS patients [75-77], and the presence of aberrant SOD1 species associated with human sporadic ALS has been extensively demonstrated [78-80]. Insoluble SOD1-containing aggregates are also a characteristic of the familial ALS linked to SOD1 mutation. In mutSOD1 transgenic mouse, the detergent-insoluble accumulation of SOD1 appears before or coincident with symptom onset [81], and in the human SOD1-associated ALS, mutant SOD1 aggregation is a pathological hallmark [82]. This feature common to familial and sporadic cases suggests a shared pathological trait.

\section{Conclusions}

Considering the few literature data about SOD1 gene regulation, it appears clear the importance of understanding how the expression of this gene can be controlled and modulated in normal and ALS pathological conditions. The molecular pathways regulating SOD1 expression at the transcriptional level have been studied, and many cis-elements and the relative trans-acting protein factors have been identified. Nevertheless, the details of most of these interactions and the in vivo consequences of their modulation for the most part are yet to be determined, mainly in relation to pathological states. Moreover, also post-transcriptional mechanisms may exert important functions in determining the global levels of functional SOD1. Indeed, the formation of specific ribonucleoprotein complexes and RNA-silencing events may provide additional mechanisms by which SOD 1 expression could be rapidly and precisely modulated. In particular, considering that microRNAs are emerging as master regulators of gene expression due to their capability to finely tune gene dosage, investigation in this field should become a preferred topic for new researches. Future gain of knowledge about these processes may help to discover previously unanticipated integrated networks, leading to new and exciting directions in the field of ALS medical research with promising prospects. Indeed, the progress in understanding the mechanisms of transcriptional and posttranscriptional control could offer hope for the development of new-generation drugs or medical treatment strategies.

\section{References}

[1] H. E. Parge, R. A. Hallewell, and J. A. Tainer, "Atomic structures of wild-type and thermostable mutant recombinant human $\mathrm{Cu}, \mathrm{Zn}$ superoxide dismutase," Proceedings of the National Academy of Sciences of the United States of America, vol. 89, no. 13, pp. 6109-6113, 1992.

[2] T. Mann and D. Kleinin, "Homocuprein and heptacuprein, coppe-protein compound of bloods and liver in mammals," Proceedings of the Royal Society B, vol. 126, pp. 303-315, 1938.

[3] J. M. McCord and I. Fridovich, "Superoxide dismutase. An enzymic function for erythrocuprein (hemocuprein)," Journal of Biological Chemistry, vol. 244, no. 22, pp. 6049-6055, 1969.

[4] I. Fridovich, "Superoxide dismutases," Annual Review of Biochemistry, vol. 44, pp. 147-159, 1975.

[5] L. Y. Chang, J. W. Slot, H. J. Geuze, and J. D. Crapo, "Molecular immunocytochemistry of the CuZn superoxide dismutase in rat hepatocytes," Journal of Cell Biology, vol. 107, no. 6, part 1, pp. 2169-2179, 1988.

[6] E. C. Chang, B. F. Crawford, Z. Hong, T. Bilinski, and D. J. Kosman, "Genetic and biochemical characterization of $\mathrm{Cu}, \mathrm{Zn}$ superoxide dismutase mutants in Saccharomyces cerevisiae," Journal of Biological Chemistry, vol. 266, no. 7, pp. 4417-4424, 1991.

[7] F. M. F. Alameddine and A. M. Zafari, "Genetic polymorphisms and oxidative stress in heart failure," Congestive Heart Failure, vol. 8, no. 3, pp. 157-172, 2002.

[8] L. W. Oberley and G. R. Buettner, "Role of superoxide dismutase in cancer: a review," Cancer Research, vol. 39, no. 4, pp. 1141-1149, 1979.

[9] M. Flekac, J. Skrha, J. Hilgertova, Z. Lacinova, and M. Jarolimkova, "Gene polymorphisms of superoxide dismutases and catalase in diabetes mellitus," BMC Medical Genetics, vol. 9, article 30, 2008.

[10] R. de la Torre, A. Casado, E. López-Fernández, D. Carrascosa, V. Ramírez, and J. Sáez, "Overexpression of copper-zinc superoxide dismutase in trisomy 21," Experientia, vol. 52, no. 9, pp. 871-873, 1996.

[11] D. R. Rosen, T. Siddique, D. Patterson et al., "Mutations in $\mathrm{Cu} / \mathrm{Zn}$ superoxide dismutase gene are associated with familial amyotrophic lateral sclerosis," Nature, vol. 362, no. 6415, pp. 59-62, 1993.

[12] H. Ohno, S. Iizuka, and T. Kondo, "The levels of superoxide dismutase, catalase, and carbonic anhydrase in erythrocytes of patients with Down's syndrome," Klinische Wochenschrift, vol. 62, no. 6, pp. 287-288, 1984.

[13] D. Levanon, J. Lieman-Hurwitz, N. Dafni et al., "Architecture and anatomy of the chromosomal locus in human chromosome 21 encoding the $\mathrm{Cu} / \mathrm{Zn}$ superoxide dismutase," The EMBO Journal, vol. 4, no. 1, pp. 77-84, 1985.

[14] S. J. Seo, H. T. Kim, G. Cho, H. M. Rho, and G. Jung, "Sp1 and C/EBP-related factor regulate the transcription of human Cu/Zn SOD gene," Gene, vol. 178, no. 1-2, pp. 177-185, 1996.

[15] H. Y. Yoo, M. S. Chang, and H. M. Rho, "Heavy metalmediated activation of the rat $\mathrm{Cu} / \mathrm{Zn}$ superoxide dismutase gene via a metal-responsive element," Molecular and General Genetics, vol. 262, no. 2, pp. 310-313, 1999. 
[16] H. Y. Yoo, M. S. Chang, and H. M. Rho, "Induction of the rat $\mathrm{Cu} / \mathrm{Zn}$ superoxide dismutase gene through the peroxisome proliferator-responsive element by arachidonic acid," Gene, vol. 234, no. 1, pp. 87-91, 1999.

[17] H. Y. Yoo, M. S. Chang, and H. M. Rho, "The activation of the rat copper/zinc superoxide dismutase gene by hydrogen peroxide through the hydrogen peroxide-responsive element and by paraquat and heat shock through the same heat shock element," Journal of Biological Chemistry, vol. 274, no. 34, pp. 23887-23892, 1999.

[18] Y. H. Kim, K. H. Park, and H. M. Rho, "Transcriptional activation of the $\mathrm{Cu}, \mathrm{Zn}$-superoxide dismutase gene through the AP2 site by ginsenoside Rb extracted from a medicinal plant, Panax ginseng," Journal of Biological Chemistry, vol. 271, no. 40 , pp. 24539-24543, 1996.

[19] L. Sherman, N. Dafni, J. Lieman Hurwitz, and Y. Groner, "Nucleotide sequence and expression of human chromosome 21-encoded superoxide dismutase mRNA," Proceedings of the National Academy of Sciences of the United States of America, vol. 80, no. 181, pp. 5465-5469, 1983.

[20] Y. Groner, J. Lieman-Hurwitz, N. Dafni et al., "Molecular structure and expression of the gene locus on chromosome 21 encoding the $\mathrm{Cu} / \mathrm{Zn}$ superoxide dismutase and its relevance to Down syndrome," Annals of the New York Academy of Sciences, vol. 450, pp. 133-156, 1985.

[21] S. J. Seo, S. S. Kang, G. Cho, H. M. Rho, and G. Jung, "C/EBP $\alpha$ and $\mathrm{C} / \mathrm{EBP} \beta$ play similar roles in the transcription of the human $\mathrm{Cu} / \mathrm{Zn}$ SOD gene," Gene, vol. 203, no. 1, pp. 11-15, 1997.

[22] T. C. Hour, Y. L. Lai, C. I. Kuan et al., "Transcriptional upregulation of SOD1 by CEBPD: a potential target for cisplatin resistant human urothelial carcinoma cells," Biochemical Pharmacology, vol. 80, no. 3, pp. 325-334, 2010.

[23] M. R. Briggs, J. T. Kadonaga, S. P. Bell, and R. Tjian, "Purification and biochemical characterization of the promoterspecific transcription factor, Sp1," Science, vol. 234, no. 4772, pp. 47-52, 1986.

[24] V. Afonso, G. Santos, P. Collin et al., "Tumor necrosis factor- $\alpha$ down-regulates human $\mathrm{Cu} / \mathrm{Zn}$ superoxide dismutase 1 promoter via JNK/AP-1 signaling pathway," Free Radical Biology and Medicine, vol. 41, no. 5, pp. 709-721, 2006.

[25] X. Cao, G. R. Guy, V. P. Sukhatme, and Y. H. Tan, "Regulation of the Egr-1 gene by tumor necrosis factor and interferons in primary human fibroblasts," Journal of Biological Chemistry, vol. 267, no. 2, pp. 1345-1349, 1992.

[26] L. R. Chaudhary, S. L. Cheng, and L. V. Avioli, "Induction of early growth response- 1 gene by interleukin- $1 \beta$ and tumor necrosis factor- $\alpha$ in normal human bone marrow stromal and osteoblastic cells: regulation by a protein kinase C inhibitor," Molecular and Cellular Biochemistry, vol. 156, no. 1, pp. 69-77, 1996.

[27] X. Zhang and Y. Liu, "Suppression of HGF receptor gene expression by oxidative stress is mediated through the interplay between Sp1 and Egr-1," American Journal of Physiology, vol. 284, no. 6, pp. F1216-F1225, 2003.

[28] N. A. Franken, R. ten Cate, C. van Bree, and J. Haveman, "Induction of the early response protein EGR-1 in human tumour cells after ionizing radiation is correlated with a reduction of repair of lethal lesions and an increase of repair of sublethal lesions," International Journal of Oncology, vol. 24, no. 4, pp. 1027-1031, 2004.

[29] I. B. Copland and M. Post, "Stretch-activated signaling pathways responsible for early response gene expression in fetal lung epithelial cells," Journal of Cellular Physiology, vol. 210, no. 1 , pp. 133-143, 2007

[30] E. Minc, P. de Coppet, P. Masson et al., "The human copperzinc superoxide dismutase gene (SOD1) proximal promoter is regulated by Sp1, Egr-1, and WT1 via non-canonical binding sites," Journal of Biological Chemistry, vol. 274, no. 1, pp. 503509, 1999.

[31] S. Baldelli, K. Aquilano, G. Rotilio, and M. R. Ciriolo, "Glutathione and copper, zinc superoxide dismutase are modulated by overexpression of neuronal nitric oxide synthase," International Journal of Biochemistry and Cell Biology, vol. 40, no. 11, pp. 2660-2670, 2008.

[32] B. K. Meyer and G. H. Perdew, "Characterization of the AhRhsp90-XAP2 core complex and the role of the immunophilinrelated protein XAP2 in AhR stabilization," Biochemistry, vol. 38, no. 28, pp. 8907-8917, 1999.

[33] A. Kazlauskas, L. Poellinger, and I. Pongratz, "Evidence that the co-chaperone $\mathrm{p} 23$ regulates ligand responsiveness of the dioxin (aryl hydrocarbon) receptor," Journal of Biological Chemistry, vol. 274, no. 19, pp. 13519-13524, 1999.

[34] J. Mimura and Y. Fujii-Kuriyama, "Functional role of AhR in the expression of toxic effects by TCDD," Biochimica et Biophysica Acta, vol. 1619, no. 3, pp. 263-268, 2003.

[35] C. L. Wilson and S. Safe, "Mechanisms of ligand-induced aryl hydrocarbon receptor-mediated biochemical and toxic responses," Toxicologic Pathology, vol. 26, no. 5, pp. 657-671, 1998.

[36] T. A. Nguyen, D. Hoivik, J. E. Lee, and S. Safe, "Interactions of nuclear receptor coactivator/corepressor proteins with the aryl hydrocarbon receptor complex," Archives of Biochemistry and Biophysics, vol. 367, no. 2, pp. 250-257, 1999.

[37] J. S. Cho, M. S. Chang, and H. M. Rho, "Transcriptional activation of the human $\mathrm{Cu} / \mathrm{Zn}$ superoxide dismutase gene by 2,3,7,8-tetrachlorodibenzo-p-dioxin through the xenobioticresponsive element," Molecular Genetics and Genomics, vol. 266, no. 1, pp. 133-141, 2001.

[38] J. Li, M. J. Calkins, D. A. Johnson, and J. A. Johnson, "Role of Nrf2-dependent ARE-driven antioxidant pathway in neuroprotection," Methods in Molecular Biology, vol. 399, pp. 67-78, 2007.

[39] W. O. Osburn and T. W. Kensler, "Nrf2 signaling: an adaptive response pathway for protection against environmental toxic insults," Mutation Research, vol. 659, no. 1-2, pp. 31-39, 2008.

[40] W. Li and A. N. Kong, "Molecular mechanisms of Nrf2mediated antioxidant response," Molecular Carcinogenesis, vol. 48, no. 2, pp. 91-104, 2009.

[41] T. Nguyen, P. Nioi, and C. B. Pickett, "The Nrf2-antioxidant response element signaling pathway and its activation by oxidative stress," Journal of Biological Chemistry, vol. 284, no. 20, pp. 13291-13295, 2009.

[42] E. Y. Park and H. M. Rho, "The transcriptional activation of the human copper/zinc superoxide dismutase gene by 2,3,7,8tetrachlorodibenzo-p-dioxin through two different regulator sites, the antioxidant responsive element and xenobiotic responsive element," Molecular and Cellular Biochemistry, vol. 240, no. 1-2, pp. 47-55, 2002.

[43] H. Dreger, K. Westphal, N. Wilck et al., "Protection of vascular cells from oxidative stress by proteasome inhibition depends on Nrf2," Cardiovascular Research, vol. 85, no. 2, pp. 395-403, 2010.

[44] E. O'Dea and A. Hoffmann, "The regulatory logic of the NFkappaB signaling system.," Cold Spring Harbor Perspectives in Biology, vol. 2, no. 1, Article ID a000216, 2010. 
[45] S. Ghosh and M. S. Hayden, "New regulators of NF- $\kappa$ B in inflammation," Nature Reviews Immunology, vol. 8, no. 11, pp. 837-848, 2008.

[46] S. Vallabhapurapu and M. Karin, "Regulation and function of NF- $\kappa \mathrm{B}$ transcription factors in the immune system," Annual Review of Immunology, vol. 27, pp. 693-733, 2009.

[47] R. Schreck, P. Rieber, and P. A. Baeuerle, "Reactive oxygen intermediates as apparently widely used messengers in the activation of the NF- $\kappa \mathrm{B}$ transcription factor and HIV-1," The EMBO Journal, vol. 10, no. 8, pp. 2247-2258, 1991.

[48] C. K. Sen and L. Packer, "Antioxidant and redox regulation of gene transcription," FASEB Journal, vol. 10, no. 7, pp. 709-720, 1996.

[49] S. K. Manna, H. J. Zhang, T. Yan, L. W. Oberley, and B. B. Aggarwal, "Overexpression of manganese superoxide dismutase suppresses tumor necrosis factor-induced apoptosis and activation of nuclear transcription factor- $\kappa \mathrm{B}$ and activated protein-1," Journal of Biological Chemistry, vol. 273, no. 21, pp. 13245-13254, 1998.

[50] N. Li and M. Karin, "Is NF- $\kappa$ B the sensor of oxidative stress?" FASEB Journal, vol. 13, no. 10, pp. 1137-1143, 1999.

[51] A. I. Rojo, M. Salinas, D. Martín, R. Perona, and A. Cuadrado, "Regulation of $\mathrm{Cu} / \mathrm{Zn}$-superoxide dismutase expression via the phosphatidylinositol 3 kinase/Akt pathway and nuclear factor- $\kappa$ B," Journal of Neuroscience, vol. 24, no. 33, pp. 73247334, 2004.

[52] T. Tagami, L. D. Madison, T. Nagaya, and J. L. Jameson, "Nuclear receptor corepressors activate rather than suppress basal transcription of genes that are negatively regulated by thyroid hormone," Molecular and Cellular Biology, vol. 17, no. 5, pp. 2642-2648, 1997.

[53] D. L. Bodenner, M. A. Mroczynski, B. D. Weintraub, S. Radovick, and F. E. Wondisford, "A detailed functional and structural analysis of a major thyroid hormone inhibitory element in the human thyrotropin $\beta$-subunit gene," Journal of Biological Chemistry, vol. 266, no. 32, pp. 21666-21673, 1991.

[54] G. M. Santos, V. Afonso, G. B. Barra et al., "Negative regulation of superoxide dismutase-1 promoter by thyroid hormone," Molecular Pharmacology, vol. 70, no. 3, pp. 793-800, 2006.

[55] C. Andreassi and A. Riccio, "To localize or not to localize: mRNA fate is in 3/UTR ends," Trends in Cell Biology, vol. 19, no. 9, pp. 465-474, 2009.

[56] A. Kilk, "Human CuZn superoxide dismutase enzymatic activity in cells is regulated by the length of the mRNA," FEBS Letters, vol. 362, no. 3, pp. 323-327, 1995.

[57] G. Shaw and R. Kamen, "A conserved AU sequence from the 3' untranslated region of GM-CSF mRNA mediates selective mRNA degradation,” Cell, vol. 46, no. 5, pp. 659-667, 1986.

[58] A. Pascale, M. Amadio, and A. Quattrone, "Defining a neuron: neuronal ELAV proteins," Cellular and Molecular Life Sciences, vol. 65, no. 1, pp. 128-140, 2008.

[59] R. S. Pillai, S. N. Bhattacharyya, and W. Filipowicz, "Repression of protein synthesis by miRNAs: how many mechanisms?" Trends in Cell Biology, vol. 17, no. 3, pp. 118-126, 2007.

[60] W. Filipowicz, S. N. Bhattacharyya, and N. Sonenberg, "Mechanisms of post-transcriptional regulation by microRNAs: are the answers in sight?" Nature Reviews Genetics, vol. 9, no. 2, pp. 102-114, 2008.

[61] Q. Wang, Y. Wang, A. W. Minto et al., "MicroRNA-377 is upregulated and can lead to increased fibronectin production in diabetic nephropathy," FASEB Journal, vol. 22, no. 12, pp. 4126-4135, 2008.
[62] T. Siddique, D. A. Fiolewicz, M. A. Pericak-Vance et al., "Linkage of a gene causing familial amyotrophic lateral sclerosis to chromosome 21 and evidence of genetic-locus heterogeneity," The New England Journal of Medicine, vol. 324, no. 20, pp. 1381-1384, 1991.

[63] H. X. Deng, A. Hentati, J. A. Tainer et al., "Amyotrophic lateral sclerosis and structural defects in $\mathrm{Cu}, \mathrm{Zn}$ superoxide dismutase," Science, vol. 261, no. 5124, pp. 1047-1051, 1993.

[64] P. M. Andersen, "Amyotrophic lateral sclerosis associated with mutations in the CuZn superoxide dismutase gene," Current Neurology and Neuroscience Reports, vol. 6, no. 1, pp. 37-46, 2006.

[65] R. Wroe, A. Wai-Ling Butler, P. M. Andersen, J. F. Powell, and A. Al-Chalabi, "ALSOD: the amyotrophic lateral sclerosis online database," Amyotrophic Lateral Sclerosis, vol. 9, no. 4, pp. 249-250, 2008.

[66] P. M. Andersen, K. B. Sims, W. W. Xin et al., "Sixteen novel mutations in the $\mathrm{Cu} / \mathrm{Zn}$ superoxide dismutase gene in amyotrophic lateral sclerosis: a decade of discoveries, defects and disputes," Amyotrophic Lateral Sclerosis and Other Motor Neuron Disorders, vol. 4, no. 2, pp. 62-73, 2003.

[67] L. Zinman, H. N. Liu, C. Sato et al., "A mechanism for low penetrance in an ALS family with a novel SOD1 deletion," Neurology, vol. 72, no. 13, pp. 1153-1159, 2009.

[68] P. Pasinelli and R. H. Brown, "Molecular biology of amyotrophic lateral sclerosis: insights from genetics," Nature Reviews Neuroscience, vol. 7, no. 9, pp. 710-723, 2006.

[69] K. Nishiyama, S. Murayama, S. Kwak, and I. Kanazawa, "Expression of the copper-zinc superoxide dismutase gene in amyotrophic lateral sclerosis," Annals of Neurology, vol. 41, no. 4, pp. 551-556, 1997.

[70] F. L. Conforti, A. Magariello, R. Mazzei et al., "Abnormally high levels of SOD1 mRNA in a patient with amyotrophic lateral sclerosis," Muscle and Nerve, vol. 29, no. 4, pp. 610-611, 2004.

[71] Y. M. Jiang, M. Yamamoto, Y. Kobayashi et al., "Gene expression profile of spinal motor neurons in sporadic amyotrophic lateral sclerosis," Annals of Neurology, vol. 57, no. 2, pp. 236251, 2005.

[72] X. S. Wang, Z. Simmons, W. Liu, P. Boyer, and J. Connor, "Differential expression of genes in amyotrophic lateral sclerosis revealed by profiling the post mortem cortex," Amyotrophic Lateral Sclerosis, vol. 7, no. 4, pp. 201-216, 2006.

[73] S. Gagliardi, E. Cova, A. Davin et al., "SOD1 mRNA expression in sporadic amyotrophic lateral sclerosis," Neurobiology of Disease, vol. 39, no. 2, pp. 198-203, 2010.

[74] E. Cova, C. Cereda, A. Galli et al., "Modified expression of Bcl-2 and SOD1 proteins in lymphocytes from sporadic ALS patients," Neuroscience Letters, vol. 399, no. 3, pp. 186-190, 2006.

[75] S. M. Chou, C. Y. Han, H. S. Wang, H. Vlassara, and R. Bucala, "A receptor for advanced glycosylation endproducts (AGEs) is colocalized with neurofilament-bound AGEs and SOD1 in motoneurons of ALS: immunohistochemical study," Journal of the Neurological Sciences, vol. 169, no. 1-2, pp. 87-92, 1999.

[76] S. Matsumoto, H. Kusaka, H. Ito, N. Shibata, T. Asayama, and T. Imai, "Sporadic amyotrophic lateral sclerosis with dementia and $\mathrm{Cu} / \mathrm{Zn}$ superoxide dismutase-positive Lewy body-like inclusions," Clinical Neuropathology, vol. 15, no. 1, pp. 41-46, 1996.

[77] N. Shibata, A. Hirano, M. Kobayashi et al., "Cu/Zn superoxide dismutase-like immunoreactivity in Lewy body-like inclusions of sporadic amyotrophic lateral sclerosis," Neuroscience Letters, vol. 179, no. 1-2, pp. 149-152, 1994. 
[78] A. Gruzman, W. L. Wood, E. Alpert et al., "Common molecular signature in SOD1 for both sporadic and familial amyotrophic lateral sclerosis," Proceedings of the National Academy of Sciences of the United States of America, vol. 104, no. 30, pp. 12524-12529, 2007.

[79] K. Forsberg, P. A. Jonsson, P. M. Andersen et al., "Novel antibodies reveal inclusions containing non-native SOD1 in sporadic ALS patients," PLoS One, vol. 5, no. 7, Article ID e11552, 2010.

[80] D. A. Bosco, G. Morfini, N. M. Karabacak et al., "Wild-type and mutant SOD1 share an aberrant conformation and a common pathogenic pathway in ALS," Nature Neuroscience, vol. 13, no. 11, pp. 1396-1403, 2010.

[81] J. D. Rothstein, "Current hypotheses for the underlying biology of amyotrophic lateral sclerosis," Annals of Neurology, vol. 65, no. 1, pp. S3-S9, 2009.

[82] M. Watanabe, M. Dykes-Hoberg, V. Cizewski Culotta, D. L. Price, P. C. Wong, and J. D. Rothstein, "Histological evidence of protein aggregation in mutant SOD1 transgenic mice and in amyotrophic lateral sclerosis neural tissues," Neurobiology of Disease, vol. 8, no. 6, pp. 933-941, 2001. 


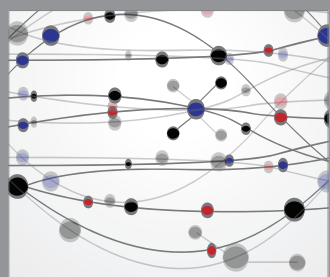

The Scientific World Journal
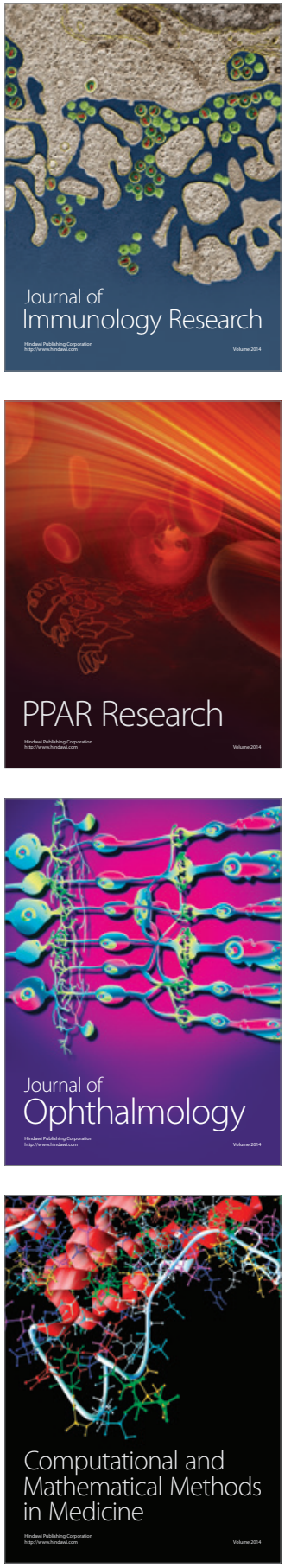

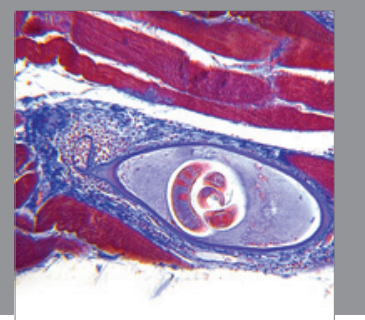

Gastroenterology

Research and Practice
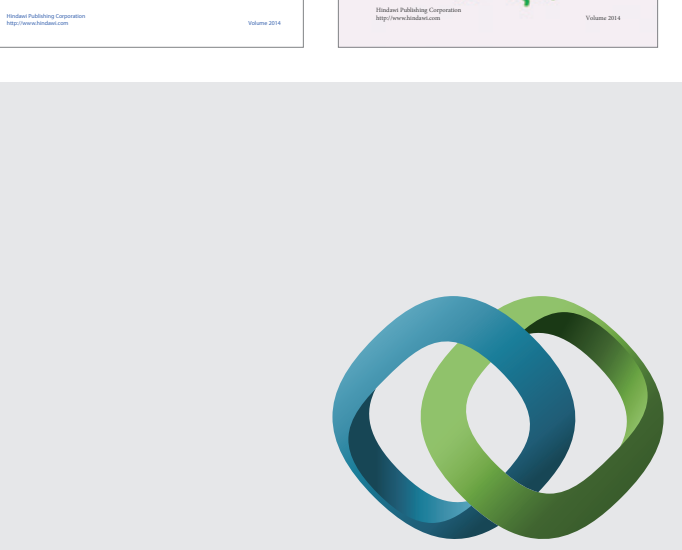

\section{Hindawi}

Submit your manuscripts at

http://www.hindawi.com
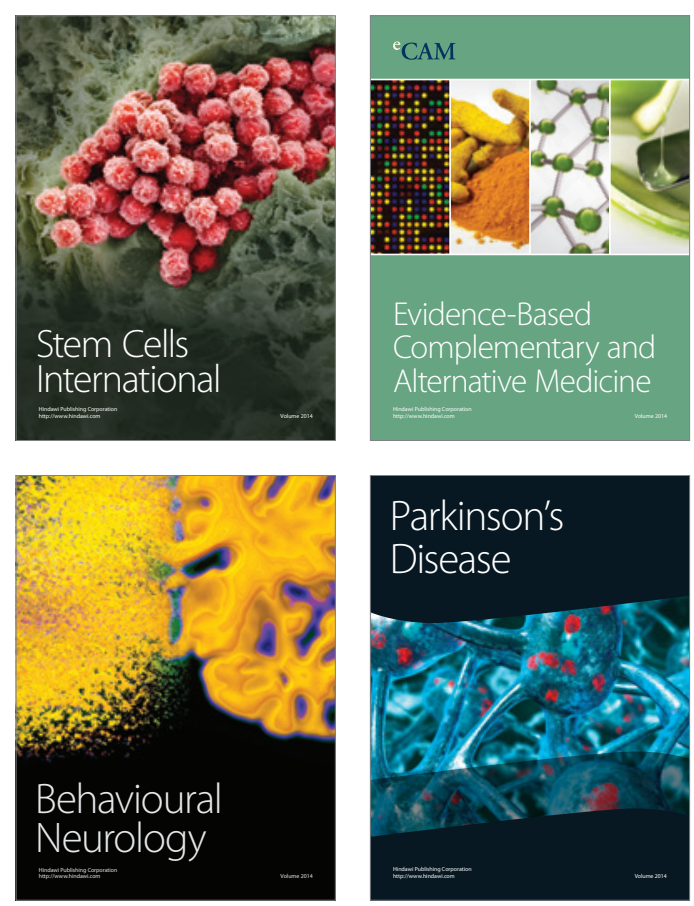

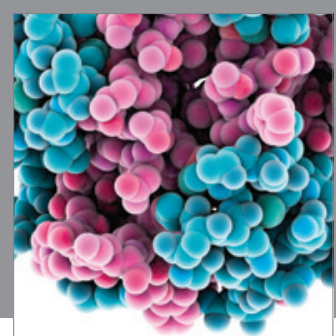

Journal of
Diabetes Research

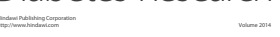

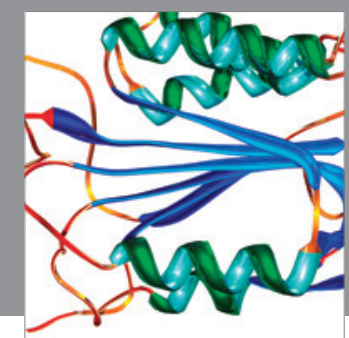

Disease Markers
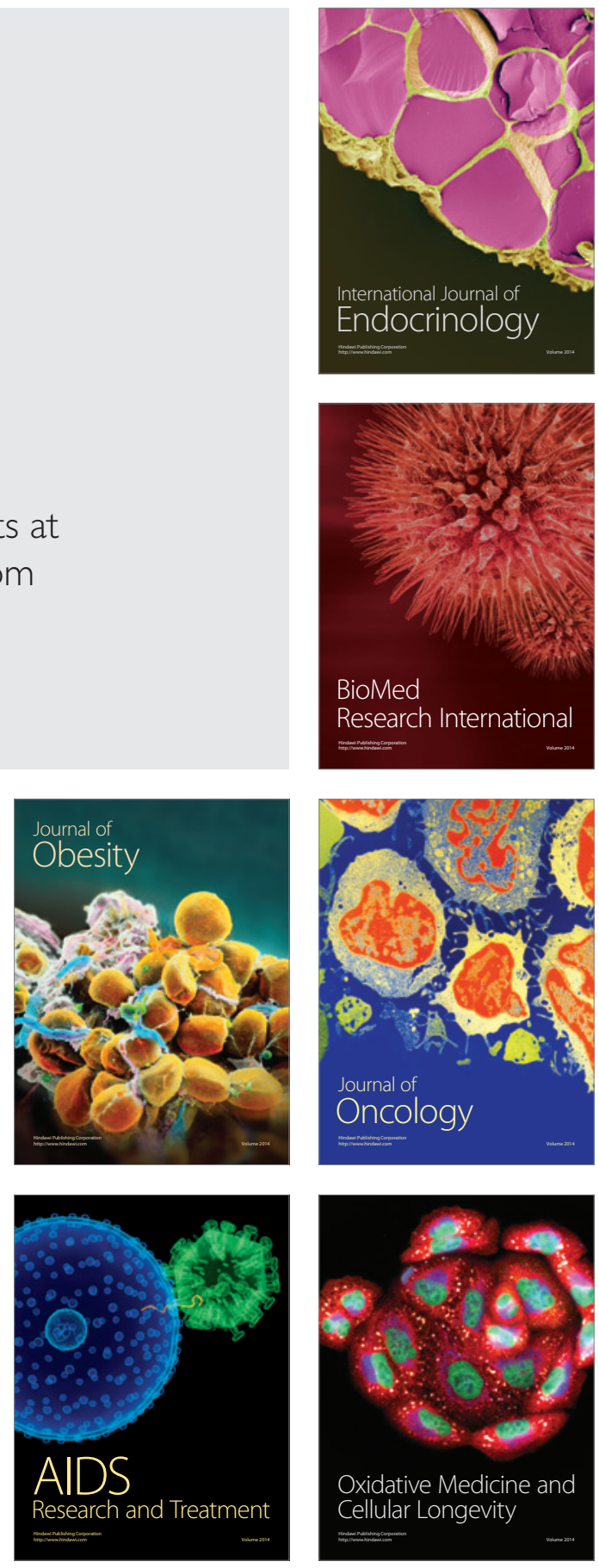